\title{
Effects of Optical Isomers of Ephedrine and Methylephedrine on the Twitch Response in the Isolated Rat Vas Deferens and the Involvement of $\alpha_{2}-$ Adrenoceptors
}

\author{
Toru Kawasuji*, Katsuo Kolke** and Haruo Saito* \\ Toyama Prefectural Institute for Pharmaceutical Research*, Toyama 939-03, \\ Japan and Department of Chemical Pharmacology, Toho University School of \\ Pharmaceutical Sciences**, Funabashi, Chiba 274, Japan
}

\begin{abstract}
The effects of optical isomers of ephedrine (EPH) and methylephedrine (MEP) on the twitch response to electrical stimulation $(1 \mathrm{msec}, 1 \mathrm{~Hz})$ in the isolated rat vas deferens were investigated to clarify the action on $\alpha_{2}$-adrenoceptors. $\quad l$-EPH $\left(10^{-7}-3 \times 10^{-5} \mathrm{M}\right)$ and $d$-EPH $\left(10^{-6}-10^{-4} \mathrm{M}\right)$ markedly inhibited the twitch response in the presence of prazosin $\left(10^{-6} \mathrm{M}\right)$. $l$-MEP also inhibited the twitch response at high concentrations $\left(3 \times 10^{-5}-10^{-3} \mathrm{M}\right)$. The rank order of inhibitory potency was $l$-EPH $>d$-EPH $\gg l$-MEP. Yohimbine $\left(3 \times 10^{-7} \mathrm{M}\right)$, a selective $\alpha_{2}$-adrenoceptor antagonist, attenuated the twitch-inhibitory effects of EPH isomers and $l$-MEP. Furthermore, the twitch-inhibitory effects of EPH isomers and $l$ MEP were attenuated by reserpine treatment $(8 \mathrm{mg} / \mathrm{kg}$, s.c.). On the other hand, $d$-MEP showed the potentiation of twitch response, and competitively antagonized the twitchinhibitory effect of clonidine $\left(10^{-9}-10^{-6} \mathrm{M}\right)$ with the $\mathrm{pA}_{2}$ value of 4.3 in the presence of prazosin. These results suggest that EPH isomers and $l$-MEP have stimulating activity for presynaptic $\alpha_{2}$-adrenoceptors. In addition, the twitch-inhibitory effect of EPH isomers and $l$-MEP may be at least partly mediated through the release of noradrenaline. It is also suggested that $d$-MEP has competitive $\alpha_{2}$-adrenoceptor antagonist activity.

Key words: Ephedrine; Methylephedrine; Optical isomer; Vas deferens; $\alpha_{2}$ Adrenoceptor
\end{abstract}

\section{Introduction}

Ephedrine and methylephedrine contain two asymmetrical carbon atoms, and then optical isomers exist. Methylephedrine, an antitussive drug, is commonly used as a racemic medicine. Ephedrine is generally accepted to have adrenaline-like activity in peripheral organs (Hoffman and Lefkowitz, 1990). Methylephedrine has been recognized as an ephedrine-like sympathomimetic amine. $\alpha$-Adrenoceptors are classified into $\alpha_{1}$ - and $\alpha_{2}$-adrenoceptors (Langer, 1981). Presynaptic $\alpha_{2}$-adrenoceptor is considered as an adrenoceptor which regulate the neurotrans-

Correspondence to: Toru Kawasuji, Toyama Prefectural Institute for Pharmaceutical Research, 17-1 Nakataikoyama, Kosugi-machi, Imizu-gun, Toyama 939-03, Japan. 
mission via inhibition of the release of neurotransmitter from nerve ending. Rat vas deferens is preferably used to investigate the effects of adrenergic drugs on $\alpha$-adrenoceptors. It is generally accepted that $\alpha$-adrenoceptor agonists produce contractions of rat vas deferens via postsynaptic $\alpha_{1}$-adrenoceptors and/or inhibit the twitch response to electrical stimulation via presynaptic inhibitory $\alpha_{2}$-adrenoceptors. In addition, it is recognized that the twitch response is at least partly mediated through the release of adenosine $5^{\prime}$-triphosphate (ATP) (Dalziel and Sneddon, 1988). Patil et al. (1967a ; 1967b) reported that $l$-and $d$-ephedrine produce contractions of rat vas deferens and the contractile activities were attenuated by reserpine treatment. These findings indicates that isomers of ephedrine have directly and indirectly stimulating activity for $\alpha_{1}$-adrenoceptors. On the other hand, the actions of optical isomers of ephedrine and methylephedrine on presynaptic $\alpha_{2}$-adrenoceptors have been uncertain (Bauer, 1981; Yamamoto et al., 1989). In the present study, effects of optical isomers of ephedrine and methylephedrine on the twitch response to electrical stimulation in isolated rat vas deferens were investigated to assess the action on $\alpha_{2}$-adrenoceptors.

\section{Methods}

Measurement of twitch response to electrical stimulation: Male Wistar strain rats, weighting 220-310 g, were killed by a blow on the head and exsanguinated. Vas deferens was isolated and immersed in oxygenated McEwen's solution. The composition of the solution was : $130 \mathrm{mM} \mathrm{NaCl}, 5.9 \mathrm{mM} \mathrm{KCl}, 2.2 \mathrm{mM} \mathrm{CaCl}_{2}, 0.9 \mathrm{mM} \mathrm{NaH}_{2} \mathrm{PO}_{4}, 25.0 \mathrm{mM} \mathrm{NaHCO}_{3}, 13.1 \mathrm{mM}$ sucrose and $11.1 \mathrm{mM}$ glucose. Because different portions of rat vas deferens are reported to show different responses to drugs (Brown et al., 1979), only the prostatic portion was used in this study. The preparation (about $1.5 \mathrm{~cm}$ ) was suspended under a resting tension of about 0.2 $\mathrm{g}$ in a $30-\mathrm{ml}$ organ bath filled with McEwen's solution, kept at $34^{\circ} \mathrm{C}$ and gassed with a mixture of $95 \% \mathrm{O}_{2}$ and $5 \% \mathrm{CO}_{2}$. The preparation was stimulated by rectangular pulses of $0.1 \mathrm{~Hz}$ in frequency, $1 \mathrm{msec}$ in duration and $65 \mathrm{~V}$, delivered by a pair of platinum ring electrodes connected to an electrostimulator (Biomedical science, Japan). The ring electrode was enveloped by polyethylene plastic except for the inside of ring. Longitudinal contractions of the preparation was recorded isometrically with an isometric transducer (TB-652T, Nihon Kohden, Japan). The preparation was allowed to equilibrate for more than $60 \mathrm{~min}$ in electrically stimulated condition. For the first $20 \mathrm{~min}$, electrical stimulation was performed at lower frequency $(0.0125$ $\mathrm{Hz}$ ). The twitch experiments were performed in the presence of $10^{-6} \mathrm{M}$ prazosin to block $\alpha_{1}-$ adrenoceptors. Prazosin was added to the bath about $30 \mathrm{~min}$ before the cumulative application of test drug. It is reported that prazosin causes the reduction of twitch response (Doxey et al., 1977). However, in the present study, the twitch response was maintained for long periods of time sufficient for cumulative application of test drugs in the presence of prazosin. This experimental advantage is considered to be at least partly due to the lack of Magnesium in McEwen's solution. The time interval between two successive concentrations of test dug was allowed to be 5 to $20 \mathrm{~min}$. The cumulative application of test drug was not repeated in a same preparation to avoid possible change in sensitivity. The ability to inhibit the twitch response caused by electrical stimulation was expressed as the pIC30 value which was a negative 
logarithm of molar concentration of test drug necessary to inhibit $30 \%$ of amplitude of twitch response. When yohimbine was used as an $\alpha_{2}$-adrenoceptor antagonist, it was added to the bath $15 \mathrm{~min}$ before drug application. To assess the involvement of endogenous noradrenaline, reserpine $(8 \mathrm{mg} / \mathrm{kg}$ ) was administered subcutaneously $24 \mathrm{hr}$ before the isolation of vas deferens.

To clarify whether antagonism is competitive or not, Schild plot analysis was performed. The $\mathrm{pA}_{2}$ value, which is the negative logarithm of dissociation constant of a competitive antagonist, was calculated according to the method of Tallarida et al. (1979), which was originally reported by Arunlakshana and Schild (1959). The concentration ratio was obtained from the ratio of IC50 values (50\% inhibitory concentrations) of clonidine in the presence and absence of test drug. In this study, the basal IC50 value in the absence of test drug was obtained from the mean curve of 5 experiments. The log (concentration ratio-1) was plotted against logarithm of molar concentration of test drug, and the regression line and its slope were calculated.

Statistical analysis: The results were expressed as the mean value \pm S.E. Statistical significance was analysed by Student's $t$-test or Welch's $t$-test after F-test. A P value less than 0.05 was considered to be significant.

Drugs: $l$-Ephedrine, $d$-ephedrine, $l$-methylephedrine and $d$-methylephedrine were kindly donated by Fuji Chemical Industries. Test drugs were used as a solution of hydrochloride salt in distilled water. Other drugs mainly used were as follows; prazosin hydrochloride, yohimbine hydrochloride and reserpine (all from Wako Pure Chemical Industries, Japan), clonidine hydrochloride (Tokyo Kasei Kogyo, Japan). These agents were used as a solution in distilled water except for prazosin and reserpine. Prazosin was suspended in ethanol, and final concentration of ethanol was less than $0.05 \%$ in the organ bath. Reserpine was used as a suspension
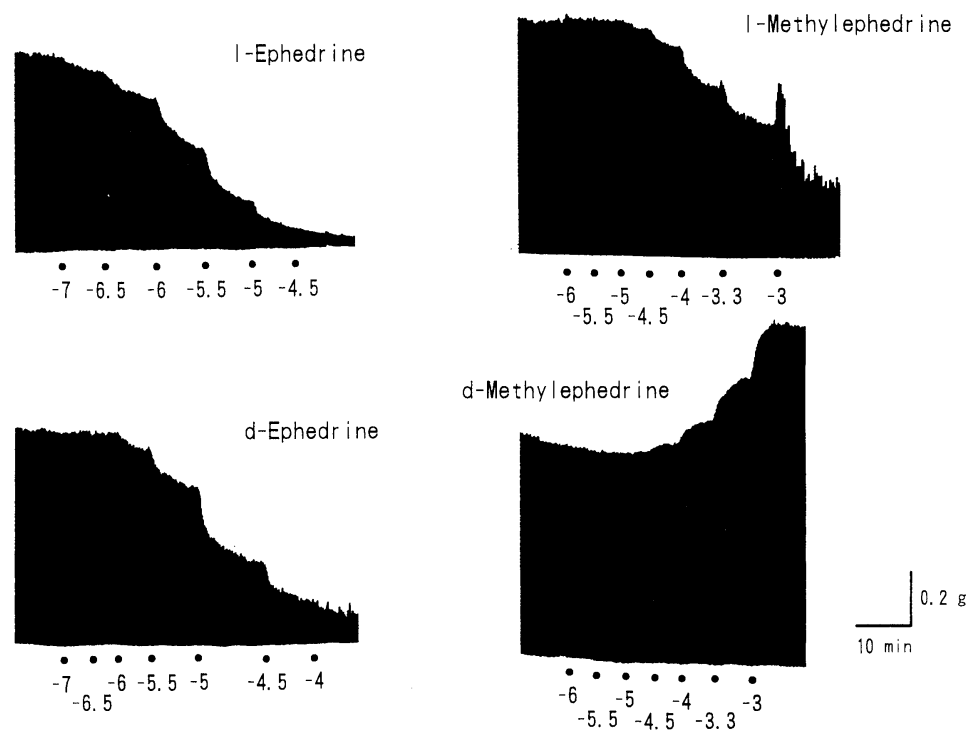

Fig. 1. Typical recording of the twitch response to electrical stimulation in the isolated rat vas deferens. Drugs were cumulatively added to the bath in the presence of $10^{-6} \mathrm{M}$ prazosin at the points indicated. For further details, see the Methods. 
in saline containing $0.5 \%$ Tween 80 (Kanto Chemical Co., Japan).

\section{Results}

In electrically stimulated rat vas deferens, $l$-ephedrine $\left(10^{-7}-3 \times 10^{-5} \mathrm{M}\right)$ and $d$-ephedrine $\left(10^{-6}-10^{-4} \mathrm{M}\right)$ markedly inhibited the twitch response in the presence of $10^{-6} \mathrm{M}$ prazosin (Figs. 1 and 2). $\quad l$-Methylephedrine $\left(3 \times 10^{-5}-10^{-3} \mathrm{M}\right)$ also inhibited the twitch response (Figs. 1 and 3$)$. On the other hand, $d$-methylephedrine showed potentiation of twitch response. The pIC30
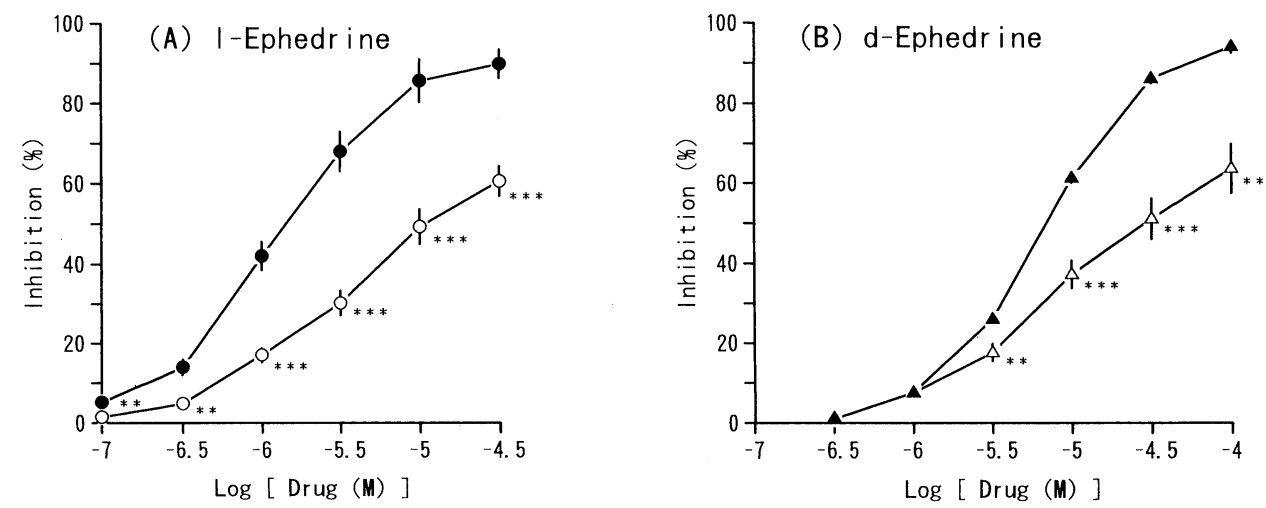

Fig. 2. Effects of $l$-ephedrine (A) and $d$-ephedrine (B) on the twitch response and the influence of yohimbine against them in the isolated rat vas deferens. Yohimbine $\left(3 \times 10^{-7} \mathrm{M}\right)$ was added 15 min before drug application. Each point is presented as a mean \pm S.E. of 5 experiments. Symbols represent: $\bullet, l$-ephedrine; $\bigcirc$, yohimbine $+l$-ephedrine; $\mathbf{\Delta}, d-$ ephedrine; $\triangle$, yohimbine $+d$-ephedrine. For further details, see the Methods. Significantly different from the value of untreated group: ${ }^{* *} \mathrm{p}<0.01$ and ${ }^{* * *} \mathrm{p}<0.001$.

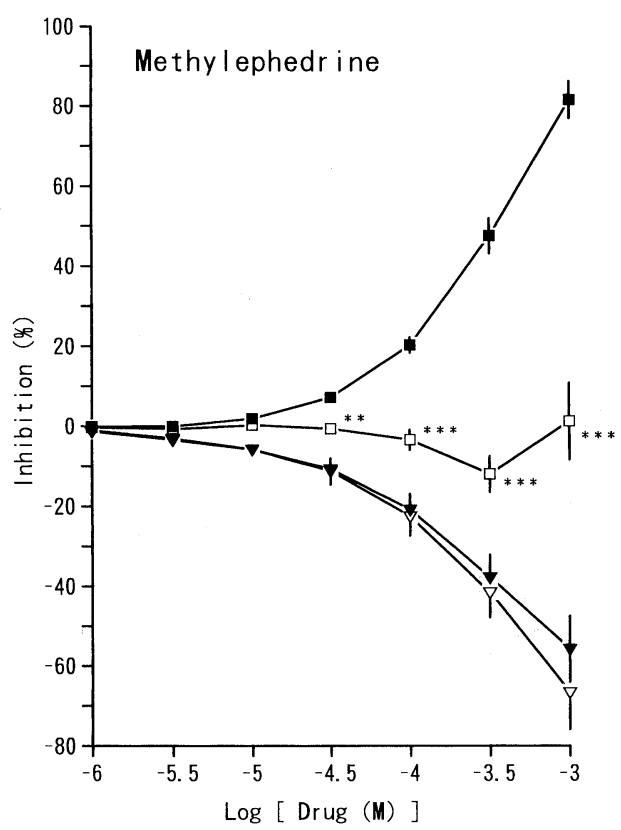

Fig. 3. Effects of optical isomers of methylephedrine on the twitch response and the influence of yohimbine against them in the isolated rat vas deferens. Yohimbine $\left(3 \times 10^{-7} \mathrm{M}\right)$ was added $15 \mathrm{~min}$ before drug application. Each point is presented as a mean \pm S.E. of 5-6 experiments. Symbols represent: $\mathbf{0}, \quad l$-methylephedrine ; $\square$, yohimbine $+l$-methylephedrine ; $\boldsymbol{\nabla}, d$-methylephedrine ; $\nabla$, yohimbine + $d$-methylephedrine. For further details, see the Methods. Significantly different from the value of untreated group : ${ }^{* *} \mathrm{p}<$ 0.01 and ${ }^{* * *} \mathrm{p}<0.001$. 

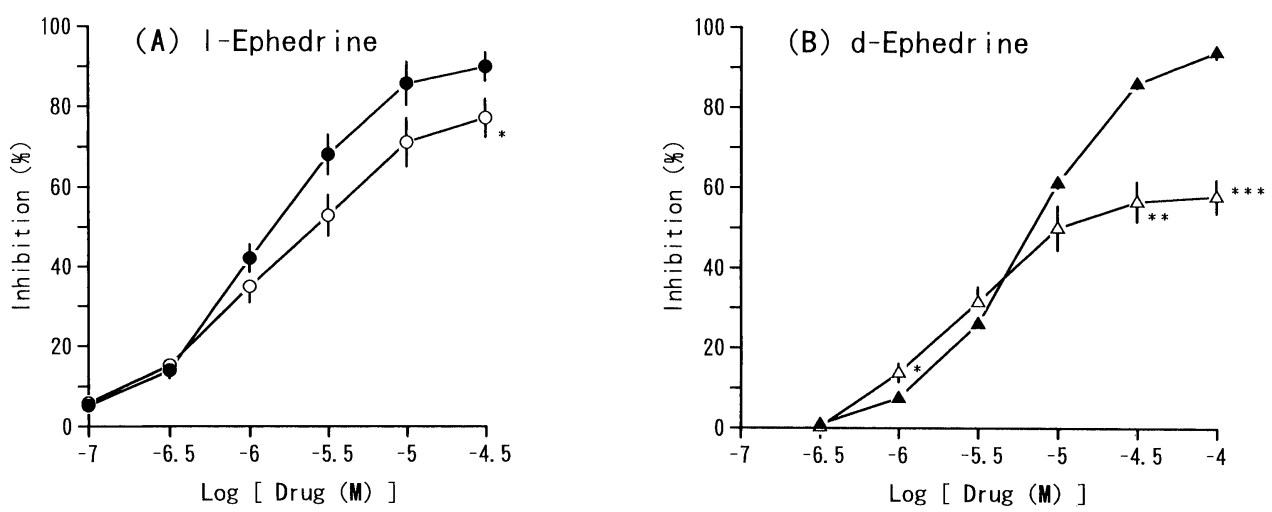

Fig. 4. Influence of reserpinization against the effects of $l$-ephedrine (A) and $d$-ephedrine (B) on the twitch response in the isolated rat vas deferens. Reserpine $(8 \mathrm{mg} / \mathrm{kg}$, s.c.) was administered $24 \mathrm{hr}$ before the isolation of vas deferens. Each point is presented as a mean \pm S.E. of 5-6 experiments. Symbols represent: $\bullet, l$-ephedrine; $\bigcirc, l$-ephedrine (reserpinized rats) ; $\mathbf{\Delta}, d$-ephedrine; $\triangle, d$-ephedrine (reserpinized rats). For further details, see the Methods. Significantly different from the value of untreated group: ${ }^{*} \mathrm{p}<0.05,{ }^{* *} \mathrm{p}<0.01$ and ${ }^{* * *} \mathrm{p}<0.001$.

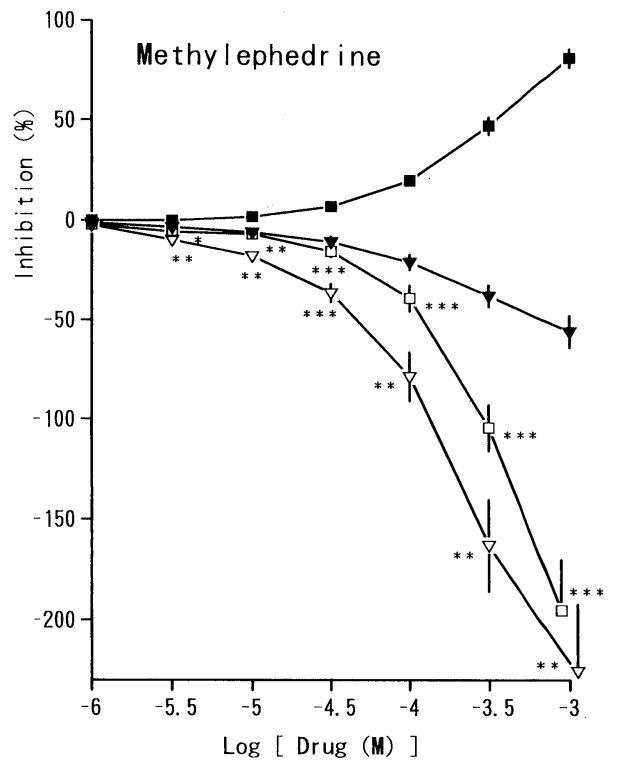

Fig. 5. Influence of reserpinization against the effects of optical isomers of methylephedrine on the twitch response in the isolated rat vas deferens. Reserpine $(8 \mathrm{mg} / \mathrm{kg}, \mathrm{s}$. c.) was administered $24 \mathrm{hr}$ before the isolation of vas deferens. Each point is presented as a mean \pm S.E. of 5-7 experiments. Symbols represent: $\mathbf{\square}, l$-methylephedrine ; $\square, \quad l$-methylephedrine (reserpinized rats); $\mathbf{\nabla}, d$-methylephedrine; $\nabla d$-methylephedrine (reserpinized rats). For further details, see the Methods. Significantly diefferent from the value of untreated group: ${ }^{*} \mathrm{p}<0.05$, ${ }^{* *} \mathrm{p}<0.01$ and ${ }^{* * *} \mathrm{p}<0.001$.

values of $l$-ephedrine, $d$-ephedrine and $l$-methylephedrine were $6.20 \pm 0.06,5.44 \pm 0.01$ and $3.82 \pm 0.04$, respectively. By pretreatment with yohimbine $\left(3 \times 10^{-7} \mathrm{M}\right)$ or by reserpinization, twitch-inhibitory effect of these drugs were attenuated or abolished (Figs. 2, 3, 4 and 5). Reserpine-induced attenuation of maximal inhibition of $d$-ephedrine was larger than that of $l$ ephedrine.

Antagonism by d-methylephedrine against the twitch-inhibitory effect of clonidine

Schild plot analysis was performed for $d$-methylephedrine which did not show the twitch- 

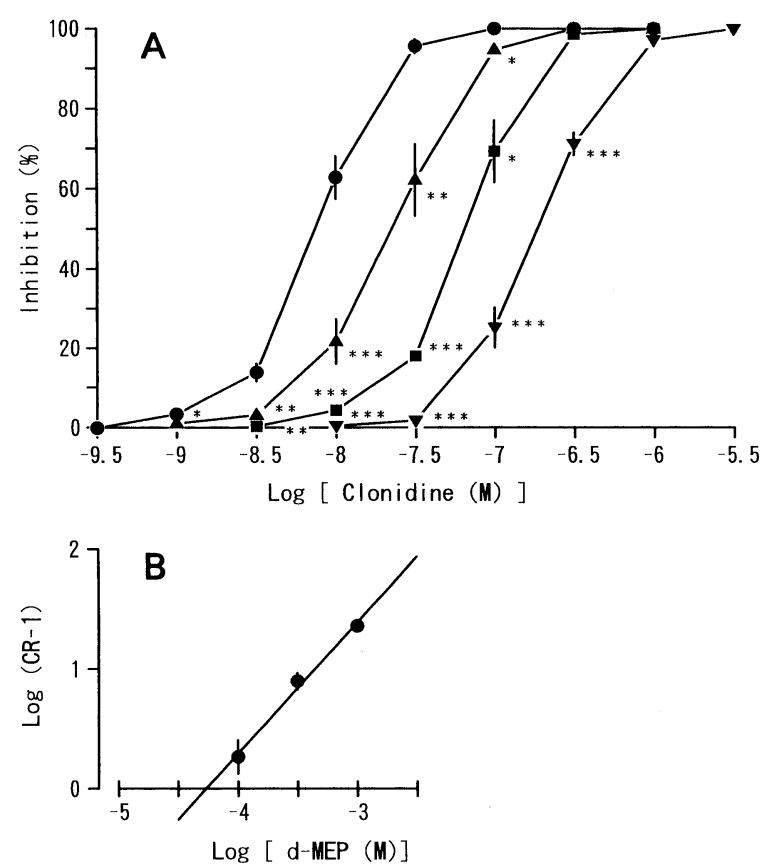

Fig. 6. Antagonism by $d$-methylephedrine against the twitch-inhibitory effect of clonidine in the isolated rat vas deferens. A : Antagonism of clonidine-induced twitch-inhibition by $d$ methylephedrine $(d-\mathrm{MEP})$. Clonidine was added cumulatively $15 \mathrm{~min}$ after drug application. Each point is presented as a mean \pm S.E. of 5 experiments. Symbols represent: control ; $\mathbf{\Delta}, d$-MEP $10^{-4} \mathrm{M} ; \mathbf{\square}, d$-MEP $3 \times 10^{-4} \mathrm{M} ; \boldsymbol{\nabla}, d$-MEP $10^{-3} \mathrm{M}$. Significantly different from the value of untreated group: ${ }^{*} \mathrm{p}<0.05,{ }^{* *} \mathrm{p}<0.01$ and ${ }^{* * *} \mathrm{p}<0.001$. B : Schild plot for antagonism of clonidine by $d$-MEP. For further details, see the Methods.

inhibitory effect. As shown in Fig. 6, the concentration-response curves of clonidine were shifted to the right in a parallel manner by $d$-methylephedrine. The slope of the regression line was not being significantly different from unity $(1.10 \pm 0.13)$. The $\mathrm{pA}_{2}$ value was $4.27 \pm 0.05$.

\section{Discussion}

$l$-Ephedrine, $d$-ephedrine and $l$-methylephedrine inhibited the twitch response in the presence of prazosin. The twitch-inhibitory effects of these drugs were attenuated by the pretreatment with yohimbine (a selective $\alpha_{2}$-adrenoceptor antagonist). Therefore, it is suggested that ephedrine isomers and $l$-methylephedrine have stimulating activity for presynaptic $\alpha_{2}$-adrenoceptors. Based on the pIC30 values, $l$-ephedrine was about 5 times and about 240 times more potent than $d$-ephedrine and $l$-methylephedrine, respectively.

It is generally accepted that ephedrine has direct and indirect stimulating action on adrenoceptors. Indirect action of sympathomimetic amine is defined as the action mediated through the release of noradrenaline from nerve endings. In the present study, the twitchinhibitory effects of ephedrine isomers and $l$-methylephedrine were attenuated or abolished by reserpinization. These results suggest that the released noradrenaline plays an important role in the twitch-inhibition by ephedrine isomers and $l$-methylephedrine. 
Phenylethylamine derivatives are reported to show weak antinociceptive action, and the mechanism of the action is suggested to be due to the $\alpha_{2}$-adrenoceptor stimulating action at least partly mediated through the release of endogenous noradrenaline in central nervous system (Matsuoka et al., 1993). Ephedrine contains the structure of phenylethylamine. From the data in the present experiments, ephedrine is considered to have $\alpha_{2}$-adrenoceptor stimulating action partly mediated through noradrenaline release. Therefore, ephedrine may produce antinociceptive action.

By addition of yohimbine, potentiation of basal twitch response was observed (data not shown). The twitch response observed in electrically stimulated vas deferens was considered to be already inhibited by $\alpha_{2}$-adrenoceptor-mediated feedback mechanism. Then, yohimbineinduced potentiation of the twitch response may be resulted from the cancellation of feedback regulation. $d$-Methylephedrine also showed the potentiation of twitch response as well as yohimbine. Then, it is thought to be possible that $d$-methylephedrine has yohimbine-like action. $\quad d$-Methylephedrine shifted concentration-response curve of clonidine to the right in a parallel manner. When Schild plot analysis was performed, the slope of regression line was not being significantly different from unity, and then the $\mathrm{pA}_{2}$ value was 4.3. From these results, it is suggested that $d$-methylephedrine has competitive and weak antagonist activity for $\alpha_{2}-$ adrenoceptors. In the vas deferens of reserpinized rat, $l$-methylephedrine showed the potentiation of twitch response as well as $d$-methylephedrine. It is considered that $l$-methylephedrine has stimulating and antagonistic activity for $\alpha_{2}$-adrenoceptors.

In the present study, $d$-methylephedrine potentiated the twitch response in reserpinized and normal vas deferens. However, the percentage of potentiation in reserpinized preparations was much larger than that in normal preparations. Reserpinization tended to lower the control twitch response which was used as $100 \%$ in calculating percent potentiation. It is suggested that the difference in percent value of potentiating effect of $d$-methylephedrine between in normal and reserpinized preparations is mainly resulted from the difference in the magnitude of control twitch response. There are several reports indicating that reserpine treatment reduce the twitch response of vas deferens (Urabe et al., 1987 : Marshall et al., 1978). It is considered that reserpine-induced depletion of tissue noradrenaline reduce noradrenalinemediated component of twitch response. In addition, it is presumed that the twitch response of reserpinized preparation may be still inhibited by the release of residual noradrenaline. These are though to be the reason why the control twitch response tended to be low in reserpinized preparations.

In the rat vas deferens, adenosine is known to inhibit the twitch response to electrical stimulation as well as ATP (Clanachan, 1977). Adenosine is a common metabolite of ATP which is released by nerve stimulation in addition with noradrenaline. It is generally accepted that indirectly acting sympathomimetic amines release catecholamines from nerve endings without exocytotic release of granular contents such as ATP (Lefkowitz et al., 1990). Therefore, it is considered that presynaptic inhibitory purinoceptors are not involved with the twitch-inhibitory effect of ephedrine isomers and $l$-methylephedrine.

In additional study, $d l$-methylephedrine (equimolar mixture of isomers) showed potentiation. The concentration-response curve was located between two curves of methylephedrine 
isomers (data not shown).

In conclusion, $l$-ephedrine, $d$-ephedrine and $l$-methylephedrine are considered to have stimulating activity for presynaptic $\alpha_{2}$-adrenoceptors in rat vas deferens. The $\alpha_{2}$-adrenoceptor agonist activity may be at least partly mediated through the release of noradrenaline. In addition, $d$-methylephedrine is suggested to have competitive antagonist activity for $\alpha_{2}-$ adrenoceptors.

\section{Acknowledgement}

We thank Fuji Chemical Industries (Japan) for the gift of optical isomers of ephedrine and methylephedrine.

\section{References}

Arunlakshana, O. and Schild, H.O. (1959). Some quantitative uses of drug antagonists. Br. J. Pharmacol. 14 : 48-58.

Bauer, V. (1981). Distribution and types of adrenoceptors in the guinea-pig ileum: the action of $\alpha$ and $\beta$-adrenoceptor agonists. Br. J. Pharmacol. 72 : 201-210.

Brown, C.M., McGrath, J.C. and Summers, R.J. (1979). The effects of $\alpha$-adrenoceptor agonists and antagonists on the responses of transmurally stimulated prostatic and epididymal portions of the isolated vas deferens of the rat. Br. J. Pharmacol. 66 : 553-564.

Clanachan, A.S., Johns, A. and Paton, D.M. (1977). Presynaptic inhibitory actions of adenine nucleotides and adenosine on neurotransmission in the rat vas deferens. Neuroscience 2: 597602.

Dalziel, H.H. and Sneddon, P. (1988). The mechanism of action of AMP-induced inhibition of sympathetic neurotransmission in the isolated vas deferens of the rat and guinea-pig. Br.J. Pharmacol. 94: 961-967.

Doxey, J.C., Smith, C.F.C. and Walker, J.M. (1977). Selectivity of blocking agents for pre- and postsynaptic $\alpha$-adrenoceptors. Br. J. Pharmacol. 60: 91-96.

Hoffman, B.B. and Lefkowitz, R.J. (1990). Catecholamines and sympathomimetic drugs. In: Goodman and Gilman's The Pharmacological Basis of Therapeutics, 8th ed., ed. by A.G. Gilman, T.W. Rall, A.S. Nies and P. Taylor, Pergamon Press, New York, pp. 187-220.

Lefkowitz, R.J., Hoffman, B.B. and Taylor, P. (1990). Neurohumoral transmission: The autonomic and somatic motor nervous systems. In: Goodman and Gilman's The Pharmacological Basis of Therapeutics, 8th ed., ed. by A.G. Gilman, T.W. Rall, A.S. Nies and P. Taylor, Pergamon Press, New York, pp. 84-121.

Langer, S.Z. (1981). Presynaptic regulation of the release of catecholamines. Pharmacol. Rev. 32 : 337-362.

Marshall, I., Nasmyth, P.A. and Shepperson, N.B. (1978). The effects of release and depletion of endogenous noradrenaline on the transmission of impulses in the mouse vas deferens. Br.J. Pharmacol. 64 : 145-152.

Matsuoka, Y., Sugioka, T., Terawaki, Y., Uruno, T. and Kubota, K. (1993). Characteristics of antinociception induced by noncatecholic phenylethylamine derivatives: the involvement of alpha-2-adrenoceptors. Japan. J. Pharmacol. 63 : 101-108.

Patil, P.N., Lapidus, J.B. and Tye, A. (1967a). Steric aspects of adrenergic drugs. I. comparative effects of DL isomers and desoxy derivatives. J. Pharmacol. Exp. Ther. 155 : 1-12. 
Patil, P.N., Lapidus, J.B., Campbell, D. and Tye, A. (1967b). Steric aspects of adrenergic drugs. II. effects of DL isomers and desoxy derivatives on the reserpine-pretreated vas deferens. J. Pharmacol. Exp. Ther. 155: 13-23.

Tallarida, R.J., Cowman, A. and Adler, M.W. (1979). $\mathrm{pA}_{2}$ and receptor differentiation : a statistical analysis of competitive antagonism. Life Sci. 25: 637-654.

Urabe, M., Kawasaki, H. and Takasaki, K. (1987). Effects of methamphetamine on the twitch response in the rat isolated vas deferens. Japan. J. Pharmacol. 45: 357-364.

Yamamoto, R., Nuki, C., Komidori, H. and Takasaki, K. (1989). Effect of $l$-ephedrine on cholinergic neurotransmission in the isolated guinea pig ileum. Japan. J. Pharmacol. 50 : 511-514.

(Received April 16, 1996 ; Accepted May 10, 1996) 